\title{
CARCINOMA PAPILÍFERO DE CISTO DO DUCTO TIREOGLOSSO: RELATO DE CASO E DISCUSSÃO SOBRE A MELHOR ESCOLHA TERAPÊUTICA
}

\author{
GOULART, Ana Paula Figueiredo Engler ${ }^{1}$ \\ SOUZA, Dandara Freitas da Silveira $\mathrm{e}^{2}$ \\ MENEZES, Felipe Hammoud de ${ }^{2}$ \\ MOREIRA, Mateus Lobato Lopes ${ }^{2}$
}

\begin{tabular}{llll}
\hline Recebido em: & 2020-04-24 & Aprovado em: 2020.05 .27 & ISSUE DOI: $10.3738 / 1982.2278 .3785$
\end{tabular}

RESUMO: Relatamos o caso clínico de um paciente de 51 anos diagnosticado com carcinoma papilífero (CP) de origem num cisto do ducto tireoglosso (CDT), identificado na peça anatomopatológica após ser submetido à cirurgia de Sistrunk. O CDT se origina durante a formação embriológica da tireoide, a partir da falha na obliteração do ducto tireoglosso. É uma lesão cística congênita, frequente na linha mediana do pescoço e raramente apresenta malignidade (menos de 1\%). O tratamento dos pacientes com carcinoma do cisto do ducto tireoglosso (CCDT) ainda é controverso. Existe debate na literatura quanto à necessidade de abordagens complementares à cirurgia de Sistrunk, como a tireoidectomia total, o esvaziamento de linfonodos cervicais, a terapia com iodo radioativo e a terapia supressiva com levotiroxina.

Palavras chave: Carcinoma do cisto do ducto tireoglosso; Carcinoma papilífero; Tireoidectomia

\section{PAPILLARY CARCINOMA OF THYROGLOSSAL DUCT CYST: CASE REPORT AND DISCUSSION OVER THE BEST THERAPEUTIC CHOICE}

SUMMARY: We report a clinical case of a 51 years old man who was diagnosed with papillary carcinoma (PC) within a thyroglossal duct cyst (TDC), identified in the anatomopathologic piece, after surgery by Sistrunk procedure. The TDC originates during the embryological formation of the thyroid, starting from obliteration failure of the thyroglossal duct. It is a common congenital cystic lesion of the midline neck and rarely malignant (less than 1\%). The treatment of patients with carcinoma of the thyroglossal duct cyst (TDCC) is still controversial. There is debate in the literature regarding the need for complementary approaches to Sistrunk procedure, such as total thyroidectomy, emptying of cervical lymph nodes, radioactive iodine therapy and suppressive therapy with levothyroxine.

Keywords: Thyroglossal Duct Cyst; Papillary Carcinoma; Thyroidectomy

\section{INTRODUÇÃO}

A glândula tireoide se origina de uma invaginação do endoderma na linha média ao nível do forame cecal durante a terceira semana de desenvolvimento fetal (ALATSAKIS, 2018). Desce do forame cecal anteriormente ao osso hioide e às cartilagens laríngeas e alcança sua posição final na frente da traqueia ao redor da sétima semana de desenvolvimento embrionário. Durante essa migração, a glândula tireoide continua ligada ao forame cecal na base da língua por meio do ducto tireoglosso, que é um trato epitelial estreito. Comumente, o ducto se desfaz entre a oitava e a décima semana de gestação, porém a falha completa no processo de obliteração do ducto pode dar origem ao cisto do ducto tireoglosso (CDT) (SUDHARSANAN,2017).

\footnotetext{
${ }^{1}$ Docente do curso de Medicina da Universidade de Franca

${ }^{2}$ Discentes do curso de Medicina da Universidade de Franca
} 
Os CDT são a anomalia mais comum no desenvolvimento da tireoide e ocorrem no osso hioide ou ao redor dele, embora menos frequentemente possam ter localização intratireoidiana ou endolaríngea (AKRAM, 2016; CASTELAN, 2017; AKRAM, 2016). Correspondem, na infância, principalmente em idade pré-escolar, a $70 \%$ das massas medianas cervicais e, nos adultos, a $7 \%$ das massas medianas cervicais, sendo mais frequentes em mulheres do que homens, na proporção 1,5:1 (ALATSAKIS,2018; STURNIOLO, 2017 WOOD,2018).

Semiologicamente, o CDT se move à deglutição e à protrusão da língua, devido à próxima relação com o osso hioide (AKRAM, 2016; SUDHARSANAN,2017). Costuma ser um nódulo esférico, móvel, liso e regular, a não ser que haja infecção, que é transmitida através do forame cecal. A localização na linha mediana e a próxima relação entre a massa cervical e o osso hioide são considerados fundamentais para o diagnóstico diferencial, que inclui cisto de fenda branquial, nódulos linfáticos, entre outros (AKRAM,2016).

A excisão cirúrgica do CDT pela técnica de Sistrunk é considerada o tratamento padrãoouro desde a sua primeira descrição no ano de 1920. Consiste em retirar todo o ducto e a porção medial do osso hioide. É apontada como a única maneira correta de remover totalmente os resquícios do ducto tireoglosso e proporcionar a diminuição das recorrências pós-cirúrgicas de cistos e fístulas do ducto tireoglosso (ALATSAKIS, 2018). Rohof et al realizaram um estudo que demonstrou que pacientes submetidos à simples excisão do cisto tiveram recorrência de 55,6\%, enquanto que pacientes submetidos à cirurgia de Sistrunk tiveram recorrência de 5,3\% (PATEL,2002; ROHOF, 2014).

Uma complicação rara é o carcinoma do CDT (CCDT), surgindo em menos de $1 \%$ dos casos (STURNIOLO, 2017; WOOD, 2018). O tipo histológico mais comum de CCDT é o carcinoma papilífero (CP), correspondendo a 75 a $90 \%$ dos casos. No entanto, menos frequentemente podem ocorrer outros tipos de carcinomas como o carcinoma misto papilífero e folicular (7\%), carcinoma de células escamosas (5\%), carcinoma folicular puro (1,7\%), carcinoma de células de Hürthle (0,6\%) e carcinoma anaplásico (0.6\%) (ALATSAKIS,2018; GORDINI 2015; MENDERICO JR.,2018). Importante ressaltar que nunca foi descrito a ocorrência do carcinoma medular (CM) em CDT (GORDINI 2015, MENDERICO JR.,2018). O $\mathrm{CM}$ é originário das células $\mathrm{C}$ ou células parafoliculares, células neuroectodérmicas cuja origem embriológica, diferentemente das células foliculares tireoidianas, é na crista neural (NILSSON,2013).

O diagnóstico do CCDT, na maioria dos casos, é feito no momento pós-operatório, pelo estudo anatomopatológico após a intervenção cirúrgica. A utilização rotineira da punção Nucleus, v.17, n.2, out.2020 
aspirativa por agulha fina (PAAF) para diagnóstico pré-operatório é incomum, devido à raridade da malignidade do CDT. Além disso, a sensibilidade da citologia é reduzida devido ao conteúdo cístico do cisto (RAYESS, 2017; TAN,2007; YANG,2000).

O tratamento dos pacientes com CCDT ainda é controverso, em parte devido à sua raridade e pela ausência de diagnóstico pré-operatório. Os estudos mais recentes indicam como primeira linha terapêutica a cirurgia de Sistrunk isoladamente, diminuindo consideravelmente as taxas de recidiva (SISTRUNK, 1920). Em contrapartida, existe um grande debate quanto à necessidade de abordagens complementares mais agressivas como a tireoidectomia total, o esvaziamento de linfonodos cervicais, a terapia com iodo radioativo e a terapia supressiva com levotiroxina. Várias publicações discutem este tema, propondo classificações de risco e algoritmos para o manejo adequado do CCDT (CHU 2002; PATEL, 2002; PERSKY 2002; WOOD, 2018).

\section{DESCRIÇÃO DO CASO: (Número de protocolo CAAE: 30941120.9.0000.5438)}

M.A.S, sexo masculino, 51 anos, tabagista (10 anos-maço), negava presença de outras comorbidades ou uso contínuo de medicamentos. Apresentou-se ao atendimento médico no ambulatório de otorrinolaringologia, com relato de nódulo na região cervical anterior, na linha mediana, acima da cartilagem tireoide, há aproximadamente 7 anos, que apresentou aumento de volume há cerca de 2 anos. No exame físico observava-se um nódulo de consistência cística, móvel à deglutição e à protrusão da língua. Com base no exame físico, suspeitou-se de CDT.

Realizou exérese do cisto no dia 08/04/2019, por meio da cirurgia de Sistrunk. O resultado anatomopatológico macroscópico confirmou a presença de CDT de 6 gramas, medindo $2,8 \times 2,3 \times 2,0 \mathrm{~cm}$, associado ao osso hioide. A análise microscópica (Quadro 1) identificou a presença de epitélio escamoso e respiratório se alinhando aos espaços císticos ao redor de tecido tireoidiano, com células foliculares apresentando núcleos grandes com fendas e pseudoinclusões (Figura 4). Esta lesão correspondia a um CP, tipo clássico, medindo 1,5 cm no maior eixo, sem invasão vascular e com margens cirúrgicas livres. O estadiamento patológico foi pT1.

Após a cirurgia de Sistrunk foi encaminhado à endocrinologia para parecer quanto à necessidade de tireoidectomia total complementar, iodo radioativo (131I) e terapia supressiva com levotiroxina. Estava bem e sem queixas. No exame físico verificou-se que a tireoide era palpável, de tamanho normal, com consistência fibroelástica e sem nodulações. Na tomografia computadorizada (TC) e ultrassonografia (USG) cervicais a glândula tireoide apresentava-se com forma, contornos, dimensões e coeficiente de atenuação normais, sem qualquer evidência de 
nódulos. A radiografia de tórax e os exames de função tireoidiana também se mostraram sem alterações.

Após revisão da literatura médica sobre o assunto, optou-se por utilizar os critérios de Wood et al (WOOD, 2018) para classificação de risco. O paciente foi classificado como de baixo risco por apresentar no CDT um CP do tipo clássico, medindo $1,5 \mathrm{~cm}$ no maior eixo, sem invasão vascular e com margens cirúrgicas livres, tireoide normal na USG e TC e sem evidência clínica ou de imagem de acometimento de linfonodos cervicais (Figura 5). Sendo assim, a cirurgia de Sistrunk isolada foi considerada suficiente no momento, e o manejo da tireoide será expectante e vigilante, com USG cervical anual, e reavaliação da conduta frente ao surgimento de lesões suspeitas.

Quadro 1 - Imagens histopatológicas e análise microscópica

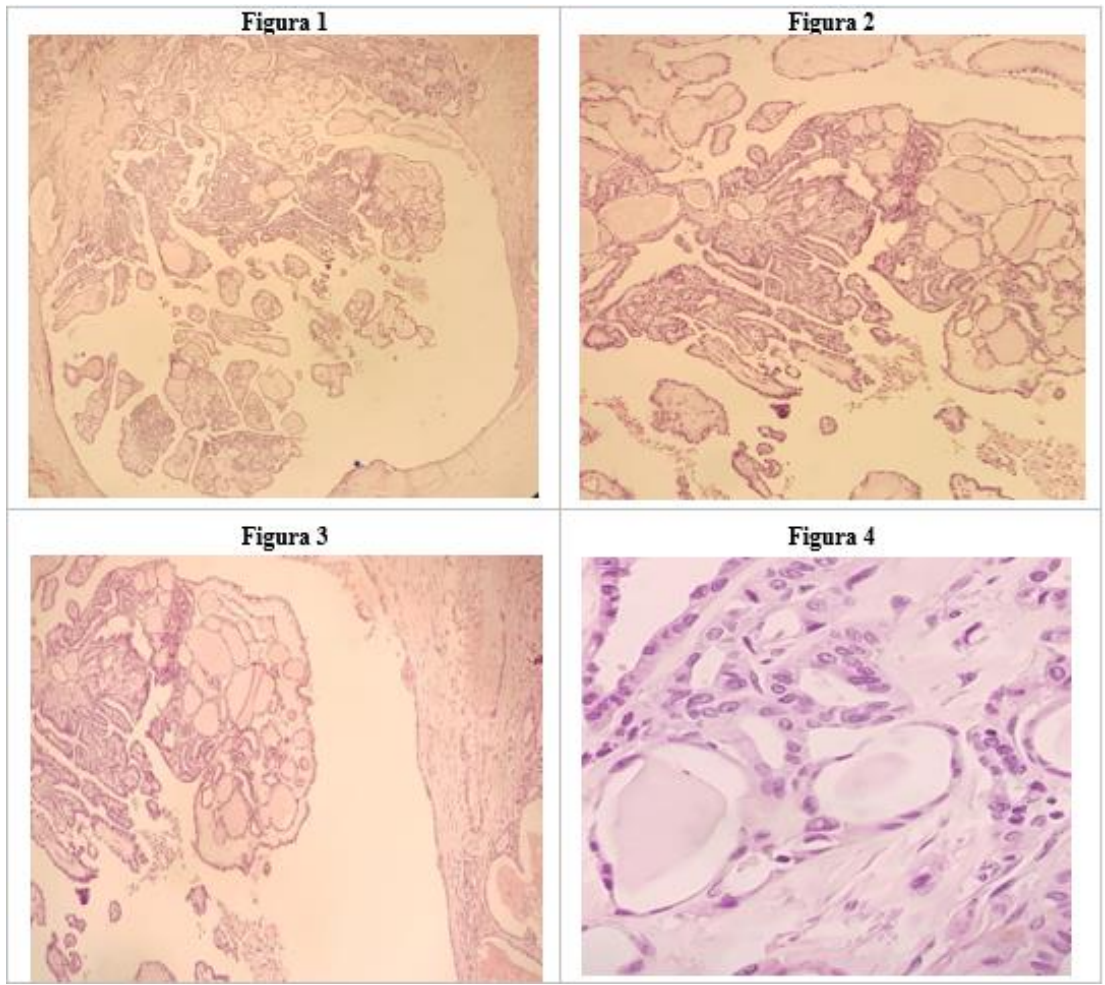

Fonte: Elaborado pelo Autor (2020)

Figura 1,2 e 3. Observam-se espaços císticos preenchidos por neoplasia de arquitetura papilar com material coloide focalmente.

Figura 4. Em grande aumento, notam-se núcleos claros com fendas e presença de pseudoinclusões.

\section{DISCUSSÃO}

O diagnóstico diferencial de massas cervicais da linha média compreende lesões congênitas, neoplásicas benignas ou malignas e inflamatórias. Como exemplo de lesões congênitas temos: CDT, tireoide ectópica, cisto dermóide e laringocele; lesões neoplásicas 
benignas ou malignas: neoplasia da tireoide, metástases de tumores de orofaringe, laringe e tireoide, lipoma, linfoma, CCDT; lesões inflamatórias: adenite bacteriana, viral ou granulomatosa (VELOSO-TELES, 2012).

Em 1911, foi relatado o primeiro caso de CCDT, sendo considerado uma entidade rara com cerca de 250 casos publicados na literatura mundial até o ano de 2017 (ALATSAKIS, 2018; STURNIOLO, 2017). A média de idade dos pacientes é próxima da quarta década de vida, similar ao caso relatado (ALATSAKIS,2018).

Em relação à origem do CCDT há atualmente duas teorias descritas na literatura. A teoria mais aceita, chamada de teoria "de novo", descreve o processo etiológico do carcinoma a partir do seu desenvolvimento primário no tecido tireoidiano ectópico presente no CDT. Corroboram essa teoria o predomínio do $\mathrm{CP}$, assim como ocorre no carcinoma primário da glândula tireoide, e o fato de não haver nenhum relato na literatura de carcinoma medular em CDT, uma vez que as células parafoliculares ou células $\mathrm{C}$ têm origem embriológica distinta (Tabela 1 e Tabela 2) (GORDINI, 2015; MENDERICO JR, 2018; STEIN 2017). Outros autores defendem a teoria metastática, segundo a qual o CCDT seria uma metástase de tumor primário presente na tireoide (GORDINI, 2015; MENDERICO JR, 2018; SUDHARSANAN, 2017).

Tabela 1 - Tipos de Carcinomas encontrados em Cistos do Ducto Tireoglosso.

\begin{tabular}{l|c}
\hline Tipo Histológico & Percentual \\
\hline Carcinoma Papilífero & 92.1 \\
\hline Carcinoma Folicular & 1.2 \\
\hline Carcinoma Escamoso & 4.3 \\
\hline Carcinoma Mucoepidermoide & 0.6 \\
\hline Carcinoma Adenoescamoso & 0.6 \\
\hline Carcinoma Papilífero e Escamoso & 1.2 \\
\hline
\end{tabular}

Fonte: Adaptado de Rayess et al (RAYESS, 2017)

Tabela 2 - Incidência relativa dos tipos de Carcinomas de Tireoide.

(Continua)

Tipo Histológico Percentual

Carcinoma Papilífero

Carcinoma Folicular

Carcinoma Medular

84

2

4

Tabela 2 - Incidência relativa dos tipos de Carcinomas de Tireoide.

(Conclusão) 
Tipo Histológico

\begin{tabular}{l|c}
\hline Carcinoma Indiferenciado & 6 \\
\hline Carcinoma Anaplásico & 1 \\
\hline Carcinoma de Células de Hürthle & 2 \\
\hline Outros & 1 \\
\hline
\end{tabular}

Fonte: Adaptado de Fagin et al (FAGIN, 2016)

O CCDT habitualmente se manifesta como uma massa cervical assintomática, como foi reportado por Rayess et al. (2017) em 95,1\% dos 164 casos analisados. A diferenciação entre CDT benigno e maligno com base em achados clínicos é extremamente difícil e a maioria dos casos são encontrados incidentalmente no estudo anatomopatológico do cisto. No entanto, podem sugerir malignidade a presença de massa de crescimento acelerado, fixa, irregular, endurecida, associada a queixas de disfonia, disfagia e perda de peso (AKRAM, 2016; ALATSAKIS, 2018; TRISTAN, 2014). O caso relatado apresentou-se como massa cervical assintomática, quadro clássico de CDT.

A USG cervical é o método de escolha na avaliação inicial das massas cervicais por ser de baixo custo, acessível, isenta de radiação e poder guiar a PAAF. O CDT geralmente se apresenta como uma imagem anecóica bem circunscrita ou pode se apresentar como uma imagem pseudo sólida, se houver conteúdo proteico no fluido do cisto (SRIVANITCHAPOOM, 2017).

A suspeita de CCDT se faz presente quando há identificação das seguintes características à USG: áreas de lesão mural na parede do cisto, com demonstração de fluxo no Doppler (HOGGINS, 2017), associadas ou não a microcalcificações; septação dentro do cisto; invasão da parede do cisto por tumor ou linfonodos cervicais suspeitos (AKRAM, 2016; SRIVANITCHAPOOM, 2017).

A PAAF é um procedimento relativamente simples, com baixa taxa de complicações que, se realizada no pré-operatório, contribui para o diagnóstico diferencial das massas cervicais, planejamento cirúrgico e aconselhamento do paciente. No entanto, a acurácia reportada da PAAF em CDT foi $53 \%$ e a taxa de exames falso negativos foi de $47 \%$, devido à hipocelularidade do conteúdo cístico (SRIVANITCHAPOOM, 2017). A realização rotineira da PAAF geralmente não é indicada tendo em vista a raridade de malignidade no CDT (cerca de 1\%). No entanto, caso a USG ou TC mostrem imagem suspeita de malignidade, faz-se necessária a PAAF, uma vez que cerca de 50\% dos casos de CCDT podem ser identificados pelo método (AKRAM, 2016).

A abordagem cirúrgica inicial é sempre o procedimento de Sistrunk. Existe falta de 
consenso na literatura sobre a necessidade de complementação com a tireoidectomia total, esvaziamento de linfonodos cervicais, complementação com ${ }^{131} \mathrm{I}$ e terapia supressiva com levotiroxina. Uma vez que CCDT é uma patologia relativamente rara, não foram conduzidos até o momento estudos clínicos randomizados ou estudos multicêntricos. Sendo assim, as recomendações da literatura são baseadas em relatos de casos e séries de casos (RAYESS, 2017).

Enquanto alguns autores consideram que o procedimento de Sistrunk seja suficiente na maioria dos casos de CCDT, outros recomendam a realização rotineira da tireoidectomia total devido à elevada incidência de $\mathrm{CP}$ ou carcinomas mistos na tireoide. A justificativa para a realização da tireoidectomia total seria a concomitância de malignidade na tireoide, a utilização do ${ }^{131}$ I como terapia adjuvante e a possibilidade de utilizar a dosagem sérica da tireoglobulina como um marcador tumoral no seguimento destes pacientes (RAYESS, 2017; ALATSAKIS, 2018).

O percentual de malignidade na tireoide é variável na literatura e abordagens cirúrgicas tendem a ser mais agressivas nos estudos cujos percentuais são mais elevados. (WOOD, 2018) Bakkar et al (2016) ( $\mathrm{n}=19)$ encontrou malignidade concomitante na tireoide em 62\%, Hartl et al (2009) ( $(=18)$ e Chrisoulidou et al (2013) $(n=6)$ também encontraram percentuais elevados: $56 \%$ e 66\%, respectivamente. Por outro lado, a série de Plaza et al (2006) (n = 5) e Miccolli et al (2004) $(n=18)$, demonstraram uma incidência menor, $20 \%$ e 33\%, respectivamente.

A revisão sistemática de Rayess et al (2017) incluiu um total de 98 artigos e um número expressivo de pacientes $(n=164)$, representando a maior revisão já feita sobre o assunto. Neste estudo, 61\% dos pacientes realizaram tireoidectomia total e destes, apenas $23,4 \%$ tiveram malignidade da tireoide concomitantemente ao CCDT. No entanto, 39\% dos pacientes analisados neste estudo não foram submetidos à tireoidectomia total. Sendo assim, considerando o número total de casos estudados, apenas $14 \%$ dos pacientes tiveram malignidade concomitante na tireoide, percentual este que tende a ter maior acurácia do que os relatados em séries com casuística reduzida.

Vale ressaltar que estudos de autópsia indicam a presença de carcinomas ocultos em até $10 \%$ dos casos, indicando que nem todos os carcinomas ocultos são clinicamente relevantes (WOOD, 2018).

A tireoidectomia total pode apresentar complicações como lesão do nervo laringeu recorrente, hipoparatireoidismo pós-cirúrgico transitório ou permanente e hematomas na incisão cirúrgica. Estudos apontam menor percentual de complicações em cirurgiões de alto volume (>100 tireoidectomias por ano). No entanto, cerca de $80 \%$ das tireoidectomias são praticadas por cirurgiões de baixo (<10 casos por ano) e médio volume (10-100 casos por ano) (FAGIN, 2016). 
Alguns autores propõem uma abordagem cirúrgica individualizada, baseada em grupos de risco. Desta maneira, o procedimento de Sistrunk isolado seria suficiente em pacientes cuja tireoide é normal clínica e radiologicamente, em pacientes de baixo risco $(<45$ anos e sem história prévia de exposição à radiação) e portadores de tumores de baixo risco $(<4 \mathrm{~cm}$, sem invasão de partes moles, sem metástases em linfonodos ou à distância e sem histologia agressiva) (RAYESS, 2017). A taxa de cura para CCDT tratado pelo procedimento Sistrunk é superior a $95 \%$ (THARMABALA, 2013).

Metástases para linfonodos cervicais são incomuns e desta maneira o esvaziamento cervical profilático não estaria indicado na ausência de linfonodos palpáveis ou linfonodos suspeitos detectados à USG (WOOD, 2018).

O uso do ${ }^{131} \mathrm{I}$ após a tireoidectomia total também é controverso. Heshmati et al (1997) e Bakkar et al (2016) consideram que o ${ }^{131} \mathrm{I}$ deve ser realizado após a tireoidectomia total somente se malignidade for encontrada na tireoide. Já Kennedy et al (1998) propôs que a tireoidectomia total sempre deve ser realizada seguida de ${ }^{131} \mathrm{I}$, independentemente da identificação de malignidade na tireoide. Rayess et al (2017) concluíram haver pouco benefício no uso do ${ }^{131}$ I no caso de o CCDT ser restrito ao cisto, sem extensão além da parede do mesmo, com margens cirúrgicas livres, sem suspeita de metástases e sem malignidade concomitante na tireoide.

Importante ressaltar que, por se tratar de patologia rara, não há estudos clínicos randomizados comparando diferentes tratamentos para o CCDT. No entanto, Wood et al (2018) propuseram um fluxograma de manejo do CCDT (Figura 5), baseado na literatura atual e na experiência do autor. Neste fluxograma, o autor classifica os pacientes entre baixo risco e alto risco dependendo de critérios anatomopatológicos do tumor no cisto. Nos casos de baixo risco, a cirurgia de Sistrunk isolada sem tireoidectomia poderia levar à cura. O autor recomenda que a possibilidade de tireoidectomia seja discutida com o paciente, uma vez que há um riso de $20 \%$ de malignidade concomitante na tireoide. Se a opção for monitorar a tireoide, os pacientes devem ser seguidos com US cervical anual. Caso o CCDT seja diagnosticado antes da cirurgia, a tireoide deve ser investigada com US e deve-se realizar PAAF de lesões suspeitas na tireoide. Uma vez confirmada malignidade concomitante na tireoide, a tireoidectomia deve ser realizada concomitante à cirurgia de Sistrunk. $\mathrm{O}$ autor considera ${ }^{131} \mathrm{I}$ caso as características do anatomopatológico do tumor da tireoide do tumor no CDT assim o justifiquem. 
Figura 5 - Estratificação de risco e decisão terapêutica.

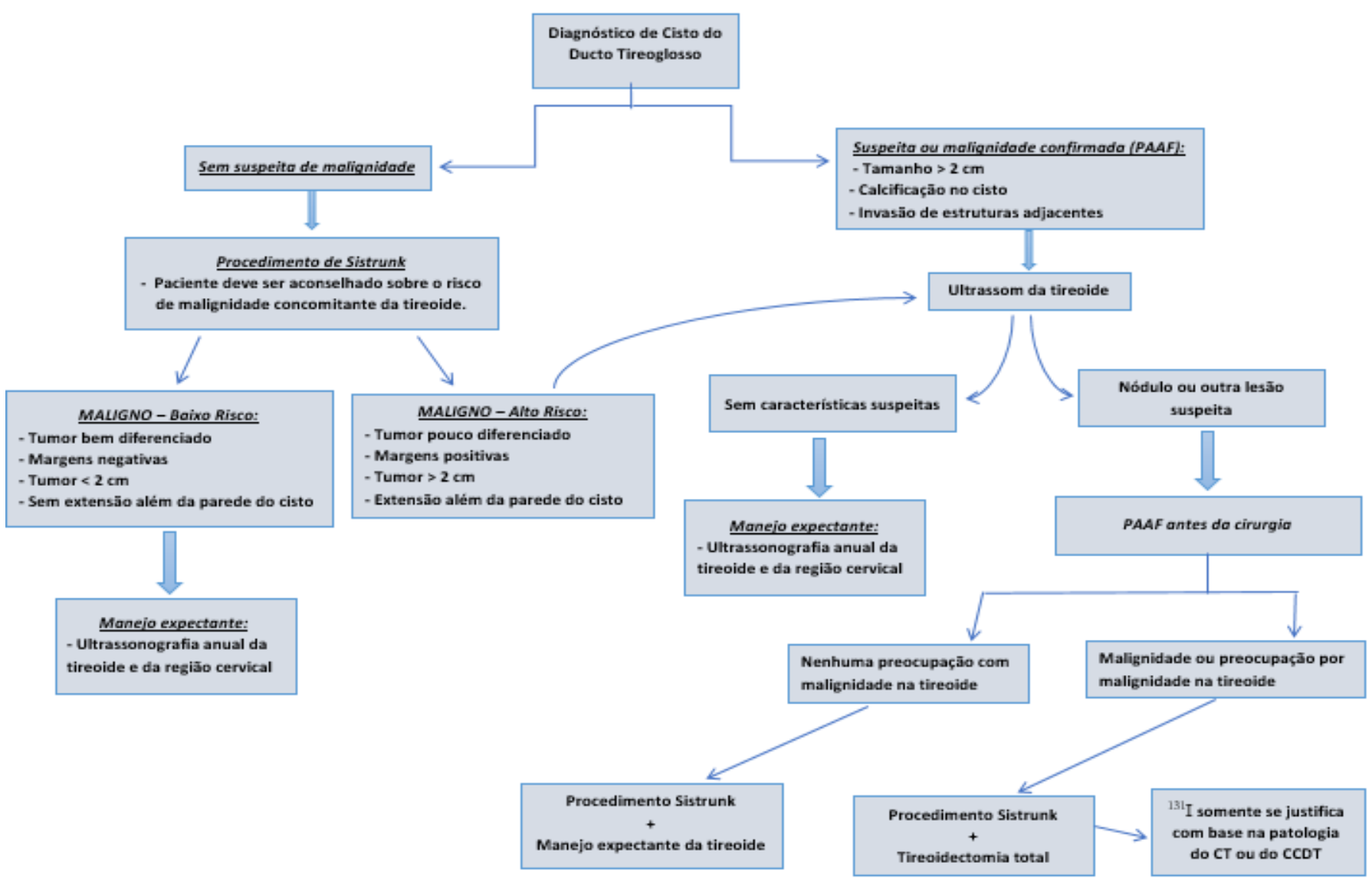

Fonte: Adaptado de Wood et al (WOOD, 2018)

PAAF: punção aspirativa por agulha fina. ${ }^{131}$ I: iodo radioativo. CT: câncer de tireoide. CCDT: carcinoma do cisto do ducto tireoglosso.

\section{CONCLUSÃO}

O CCDT é uma patologia rara que deve ser considerada no diagnóstico diferencial de massas cervicais da linha média. Na maioria dos casos, o diagnóstico é feito a partir do estudo anatomopatológico após intervenção cirúrgica no CDT. Os estudos até o momento consistem em relatos de casos, séries de casos e revisões sistemáticas.

Não há estudos clínicos randomizados comparando diferentes tratamentos para o CCDT que forneçam diretrizes baseadas em evidências e ainda há controvérsia quanto à extensão do tratamento cirúrgico e necessidade de tratamento complementar como o ${ }^{131}$ I. Porém, segundo a literatura, o tratamento padrão é a cirurgia de Sistrunk, geralmente curativa nos casos de baixo risco. Procedimentos mais invasivos, como a tireoidectomia total e o ${ }^{131} \mathrm{I}$, costumam ser indicados em casos de alto risco com malignidade na tireoide comprovada, denotando a importância de se identificar com segurança os pacientes de alto risco.

Baseado na literatura atual e na experiência do autor, Wood et al. (2018) propuseram uma padronização do tratamento através de um fluxograma que classifica o risco e direciona a 
terapêutica. Os casos de baixo risco, como o caso relatado, seriam tratados de maneira conservadora com a cirurgia de Sistrunk seguida de vigilância contínua da tireoide com US.

\section{REFERÊNCIAS}

AKRAM, Rahim et al. Adult Thyroglossal Duct Carcinoma of Thyroid Epithelial Origin: a retrospective observational study. Indian Journal Of Otolaryngology And Head \& Neck Surgery, [s.1.], v. 68, n. 4, p. 522-527, 13 set. 2016. Springer Science and Business Media LLC.

ALATSAKIS, Michael et al. Invasive Thyroglossal Duct Cyst Papillary Carcinoma: a case report and review of the literature. American Journal Of Case Reports, [s.1.], v. 19, p. 757-762, 28 jun. 2018. International Scientific Information, Inc..

BAKKAR, Sohail et al. The extent of surgery in thyroglossal cyst carcinoma. Langenbeck's Archives Of Surgery, [s.1.], v. 402, n. 5, p. 799-804, 23 jun. 2016. Springer Science and Business Media LLC.

CASTELAN, João de Bona et al. Cisto Ducto Tireoglosso: Relato de caso e revisão de literatura. Arquivos Catarinenses de Medicina, [S.1.], v. 46, n. 1, p. 125-129, jul. 2017.

CHRISOULIDOU, Alexandra et al. Thyroglossal duct cyst carcinomas: is there a need for thyroidectomy? Hormones, [s.1.], v. 12, n. 4, p. 522-528, 15 out. 2013. Springer Science and Business Media LLC.

CHU, Young Chae et al. Primary Papillary Carcinoma Arising in a Thyroglossal Duct Cyst. Yonsei Medical Journal, [s.1.], v. 43, n. 3, p. 381-384, 2002. Yonsei University College of Medicine (KAMJE).

FAGIN, James A. et al. Biologic and Clinical Perspectives on Thyroid Cancer. New England Journal Of Medicine, [s.1.], v. 375, n. 11, p. 1054-1067, 15 set. 2016. Massachusetts Medical Society.

GORDINI, Luca et al. Tall cell carcinoma arising in a thyroglossal duct cyst: a case report. Annals Of Medicine And Surgery, [s.1.], v. 4, n. 2, p. 129-132, jun. 2015. Elsevier BV.

HARTL, Dana M. et al. High Rate of Multifocality and Occult Lymph Node Metastases in Papillary Thyroid Carcinoma Arising in Thyroglossal Duct Cysts. Annals Of Surgical Oncology, [s.1.], v. 16, n. 9, p. 2595-2601, 30 jun. 2009. Springer Science and Business Media LLC.

HESHMATI, Hassan M. et al. Thyroglossal Duct Carcinoma: report of 12 cases. Mayo Clinic Proceedings, [s.1.], v. 72, n. 4, p. 315-319, abr. 1997. Elsevier BV.

HIGGINS, Jason J. et al. Papillary Carcinoma Arising in a Thyroglossal Duct Cyst. Ultrasound Quarterly, [s.1.], v. 33, n. 2, p. 179-180, jun. 2017. Ovid Technologies (Wolters Kluwer Health).

KENNEDY, Thomas L. et al. Thyroglossal duct carcinoma: a rational approach to management. The Laryngoscope, [s.1.], v. 108, n. 8, p. 1154-1158, ago. 1998. Wiley. 
MENDERICO JR, Gilberto et al. Carcinoma papilífero de cisto de ducto tireoglosso. Revista Brasileira de Cirurgia da Cabeça e Pescoço. v.46, n.1, p. 39-42. 2017.

MICCOLI, Paolo et al. Extent of Surgery in Thyroglossal Duct Carcinoma: reflections on a series of eighteen cases. Thyroid, [s.1.], v. 14, n. 2, p. 121-123, fev. 2004. Mary Ann Liebert Inc.

NILSSON, Mikael et al. Mechanisms of Thyroid Development and Dysgenesis. Current Topics In Developmental Biology, [s.1.], p. 123-170, 2013. Elsevier.

PATEL, Snehal G. et al. Management of well-differentiated thyroid carcinoma presenting within a thyroglossal duct cyst. Journal Of Surgical Oncology, [s.l.], v. 79, n. 3, p. 134-139, 21 fev. 2002. Wiley.

PERSKY, Mark S. et al. Total Thyroidectomy as Appropriate Treatment for Papillary Carcinoma in a Thyroglossal Duct Cyst. Archives Of Otolaryngology-head \& Neck Surgery, [s.1.], v. 128, n. 4, p. 463, 1 abr. 2002. American Medical Association (AMA).

RAYESS, Hani M. et al. Thyroglossal Duct Cyst Carcinoma: a systematic review of clinical features and outcomes. Otolaryngology-head And Neck Surgery, [s.1.], v. 156, n. 5, p. 794-802, 21 mar. 2017. SAGE Publications.

ROHOF, Daan et al. Recurrences after thyroglossal duct cyst surgery: results in 207 consecutive cases and review of the literature. Head \& Neck, [s.1.], v. 37, n. 12, p. 1699-1704, 25 set. 2014. Wiley.

SISTRUNK, Walter Ellis et al. THE SURGICAL TREATMENT OF CYSTS OF THE THYROGLOSSAL TRACT. Annals Of Surgery, [s.1.], v. 71, n. 2, p. 121-122, fev. 1920. Ovid Technologies (Wolters Kluwer Health).

STEIN, Tracy et al. Can Medullary Thyroid Carcinoma Arise in Thyroglossal Duct Cysts? A Search for Parafollicular C-cells in 41 Resected Cases. Head And Neck Pathology, [s.1.], v. 12, n. 1, p. 71-74, 23 maio 2017. Springer Science and Business Media LLC.

STURNIOLO, Giacomo et al. Thyroid cancer in lingual thyroid and thyroglossal duct cyst. Endocrinología, Diabetes y Nutrición, [s.1.], v. 64, n. 1, p. 40-43, jan. 2017. Elsevier BV.

SRIVANITCHAPOOM, Chonticha et al. Ultrasonographic findings of thyroglossal duct papillary carcinoma: a case report. International Journal Of Surgery Case Reports, [s.1.], v. 32, p. 54-57, 2017. Elsevier BV.

SUDHARSANAN, Sundaramurthi et al. A Rare Case of Carcinoma in the Thyroglossal Duct Cyst of an Elderly Patient. Cureus, [s.1.], p. 1-2, 19 jun. 2017. Cureus, Inc..

TAN, Geok Chin et al. Papillary carcinoma of the thyroglossal duct cyst in a 15-year-old girl. International Journal Of Pediatric Otorhinolaryngology Extra, [s.1.], v. 2, n. 2, p. 72-75, jun. 2007. Elsevier BV. 
THARMABALA, Mehala et al. Incidental thyroid papillary carcinoma in a thyroglossal duct cyst - management dilemmas. International Journal Of Surgery Case Reports, [s.1.], v. 4, n. 1, p. 58-61, 2013. Elsevier BV.

TRISTAN, Julie de et al. Thyroglossal duct cysts: 20 years' experience (1992-2011). European Archives Of Oto-rhino-laryngology, [s.1.], v. 272, n. 9, p. 2513-2519, 19 ago. 2014. Springer Science and Business Media LLC.

VELOSO-TELES, Rafaela et al. Carcinoma papilar primário num quisto do canal tireoglosso Caso clínico. Revista portuguesa de Otorrinolaringologia e Cirurgia cérvico-facial, [s.1.], v. 50, n. 1, p.59-63, march 2012.

WOOD, C. Burton et al. Papillary-Type Carcinoma of the Thyroglossal Duct Cyst: the case for conservative management. Annals Of Otology, Rhinology \& Laryngology, [s.1.], v. 127, n. 10, p. 710-716, 9 ago. 2018. SAGE Publications.

YANG, Yi Jun et al. Diagnosis of papillary carcinoma in a thyroglossal duct cyst by fine-needle aspiration biopsy. Arch Patol Lab Med, [s.1.], v. 124, p.139-142, 2000. 